\title{
Evaluation of debris mitigation options for a large constellation
}

\author{
Hugh G. Lewis \\ University of Southampton, Astronautics Research Group, Faculty of Engineering and Physical Sciences, \\ Boldrewood Innovation Campus 176/2041, Southampton SO16 7QF, UK, hglewis@soton.ac.uk
}

\begin{abstract}
Large constellations of satellites in Low Earth Orbit (LEO) may adversely impact the sustainable use of the space environment over the long-term unless appropriate plans for orbital debris mitigation measures are incorporated into the design and operation. In particular, recent computer modelling studies have shown that comprehensive observance of the Inter-Agency Space Debris Coordination Committee (IADC) guideline on post-mission disposal is a vital element of debris mitigation plans intended for large constellations in LEO. Several operators have proposed that the region of LEO below $600 \mathrm{~km}$ altitude could be used to accommodate large numbers of spacecraft without unduly heightening orbital debris concerns. The objective of this study, using the DAMAGE computer model, was to evaluate the implications for the orbital debris population of orbit design options for a large constellation. The build-up, replenishment and disposal of a large, complex constellation comprising 10,440 active spacecraft was simulated. A subset of the constellation, a component comprising 1800 spacecraft in a single band, was deployed at either $1100 \mathrm{~km}$ or $550 \mathrm{~km}$ altitude. The positioning of this component provided the two fundamental study conditions. The results showed that positioning the 1800-spacecraft component at $550 \mathrm{~km}$ altitude reduced the overall impact of the constellation substantially, whilst also reducing the need for a high post-mission disposal success rate.
\end{abstract}

\section{INTRODUCTION}

Large constellations of satellites in Low Earth Orbit (LEO) aim to provide important communications and broadband internet services to large parts of the world where the necessary terrestrial infrastructure is lacking. It is also possible for these constellations to have a significant effect on the space environment and other space users unless appropriate space debris mitigation measures are incorporated into the design and operation of the constellations. Attempts have been made to understand these impacts using space debris evolutionary models [1-5], but the complexity of the possible constellation designs means that a complete understanding is difficult to achieve; some constellation design decisions could result in significant impacts whilst others may provide ways to mitigate unwanted impacts.

In general, results reported by [1] and [2] for a large constellation at $1100 \mathrm{~km}$ altitude indicated that the impact of the constellation on the LEO orbital object population could be separated into three phases:

- a quick population rise during the constellation build-up and replenishment;

- a period of population decay as post-mission disposal (PMD) activity reduced the number of constellation satellites; and

- a long-term, gradual increase in the population due to collisions involving failed constellation satellites.

The successful post-mission disposal of spacecraft making up the constellation provides the key debris mitigation measure, but this places a demanding requirement on the spacecraft design and manufacture to achieve a high reliability of the spacecraft subsystems, especially those associated with the post-mission disposal.

In a recent study, the US National Aeronautics and Space Administration (NASA) [6] indicated that a 99\% postmission disposal reliability is needed "to mitigate the serious long-term debris generation potential." However, the results from [2] suggest that constellation spacecraft maneuvering successfully to disposal orbits with reduced lifetimes will be responsible ultimately for elevating the likelihood of collisions below the mission altitude. An increase in the post-mission success rate produces a corresponding increase in the number of spacecraft on disposal orbits and, hence, the number of conjunctions and collisions below the mission altitude. The impact of these decaying spacecraft on the orbital debris population can be reduced if the residual orbital lifetimes of the retired constellation spacecraft are decreased by maneuvering to orbits with perigee altitudes below $400 \mathrm{~km}$ [2]. 
Nevertheless, this again places a burden on the spacecraft subsystems and increases the effort needed to manage the trajectory of each spacecraft safely.

An option to reduce the collision threat from failed spacecraft is to separate the orbital planes of the constellation, such that each plane is at a different altitude and at a different orbital inclination (to maintain a consistent precession rate). The separation in these two orbital parameters reduces the probability that the orbits of failed spacecraft from one plane intersect the orbits of active or failed spacecraft from another plane [7]. Whilst this does not reduce the need for operators to aim for a high reliability of their spacecraft, it does address some concerns over the long-term presence of these failed spacecraft in the LEO region.

Finally, several operators have now proposed that the region of LEO below $600 \mathrm{~km}$ altitude could be used to accommodate large constellations of spacecraft without unduly heightening orbital debris concerns. At these low altitudes the atmospheric density is such that spacecraft can decay within a few years, thereby meeting the expectation that they will be removed from the LEO region within 25 years even if the spacecraft were to fail. Quite recently, SpaceX re-designed the Starlink constellation to move a large proportion of its spacecraft from $1100 \mathrm{~km}$ to $550 \mathrm{~km}$ altitude in a measure partly used to reduce the impact on the environment.

In an effort to further understanding of the potential impacts of large constellations on the space environment, the study reported in this paper aimed to assess the potential impacts on the LEO space environment of deploying a large constellation, comprising 10,440 spacecraft. Additionally, the study was designed to evaluate mitigation measures based on variations in the spacecraft orbits, including the separation of orbital planes and the move of a proportion of spacecraft from a relatively high altitude to a lower altitude.

\section{SIMULATION APPROACH}

DAMAGE is a high-fidelity three-dimensional physical model capable of simulating the evolution of future debris populations. The process used in DAMAGE to build and subsequently replenish constellations is based on a launch schedule comprising the number of launches per year, the number of satellites on each launcher, and the duration over which the build and replenishment are to take place. If an electric propulsion option is selected, a low altitude deployment from the launcher can be specified and the DAMAGE orbital propagator will compute the ascent trajectories for the constellation satellites, incorporating a user-specified ascent time. Throughout this period, the user can indicate whether the satellites are capable of collision avoidance. Satellites launched via the replenishment schedule replace the corresponding satellites in the constellation, and the older satellites are retired even if they have not reached the end of their service lifetime, following the user-specified post-mission disposal behavior. Once in service, the satellites maintain their within-plane spacing and inter-plane spacing subject only to Earth zonal gravity perturbations.

The Concepts of Operations (ConOps) for all spacecraft in the simulation are constructed from a set of waypoints, which identify orbital elements and times from orbital injection through to passivation (assuming the spacecraft remains under active control throughout). For spacecraft in the large constellations simulated in this study, the ConOps featured low-thrust maneuvers and a post-mission disposal process divided into two distinct elements, all encapsulated by six waypoints for the period when the spacecraft was under active control (e.g. see Fig. 1).

DAMAGE is able to simulate the deployment of constellation satellites into orbital planes that are separated by a user-specified altitude [8]. To ensure that the orbital precession induced by the Earth zonal gravity perturbations affect the orbital planes in the constellation equally, thus maintaining the constellation geometry, the inclination of each plane is computed depending on the size of its semi-major axis.

The basic simulation parameters used for this study correspond to the parameters used for the current reference case adopted by the IADC:

- A 1 February 2018 epoch with an initial population corresponding to all objects $\geq 10 \mathrm{~cm}$ residing within or crossing the LEO protected region;

- $\quad$ Launch traffic was assumed to be represented by the repetition of recent launches (taken from 1 January 2010 to 31 December 2017) with small random adjustments made to the exact launch date and orbital parameters to avoid artificially enhancing the likelihood of collisions on launch;

- New spacecraft and rocket upper stages in the non-constellation traffic were assumed to achieve a $90 \%$ success rate with respect to post-mission disposal, targeting an uncontrolled re-entry within 25 years. No collision avoidance maneuvers were implemented. 
- Vehicle passivation was assumed to be $100 \%$ successful such that no explosions were permitted within the projection period.

A large constellation was added to this reference case, with the build-up phase commencing on 1 January 2020 and the constellation ending operations on 1 January 2065. The constellation was assumed to consist of 10,440 spacecraft in seven discrete components, as detailed in Tab. 1. These seven components appeared in three altitude regimes: Very Low Earth Orbit (VLEO; 345-355 km altitude), Medium Low Earth Orbit (MLEO; 1100 km or 550 km altitude) and High Low Earth Orbit (HLEO; 1150-1250 km altitude). The design of each of these components was consistent with a Walker-Delta pattern, with all spacecraft injected into a circular orbit at an altitude of $345 \mathrm{~km}$ and then ascending to their respective mission altitudes after a 10-day checkout period. The MLEO component was assessed at mission altitudes of $1100 \mathrm{~km}$ and $550 \mathrm{~km}$ (with a corresponding change to the disposal staging altitude) to understand the effect of this design change on the orbital debris population. Every constellation spacecraft was assumed to have a mass of $250 \mathrm{~kg}$, a cross-sectional area (for atmospheric drag and collision probability estimation) of 12 square meters and a maximum lifetime of 9 years. In general, it was assumed that the post-mission disposal success rate for all constellation spacecraft was $95 \%$, collision avoidance was $100 \%$ successful throughout all phases of the mission when the spacecraft were active (i.e. including the descent from the mission altitude for disposal), and rocket stages used to deploy the spacecraft were de-orbited immediately. The spacecraft disposal was separated into two stages: an initial descent to a circular staging altitude followed by the lowering of the perigee altitude to achieve an eccentric orbit with the perigee at an altitude of $300 \mathrm{~km}$ and the apogee at the staging altitude.

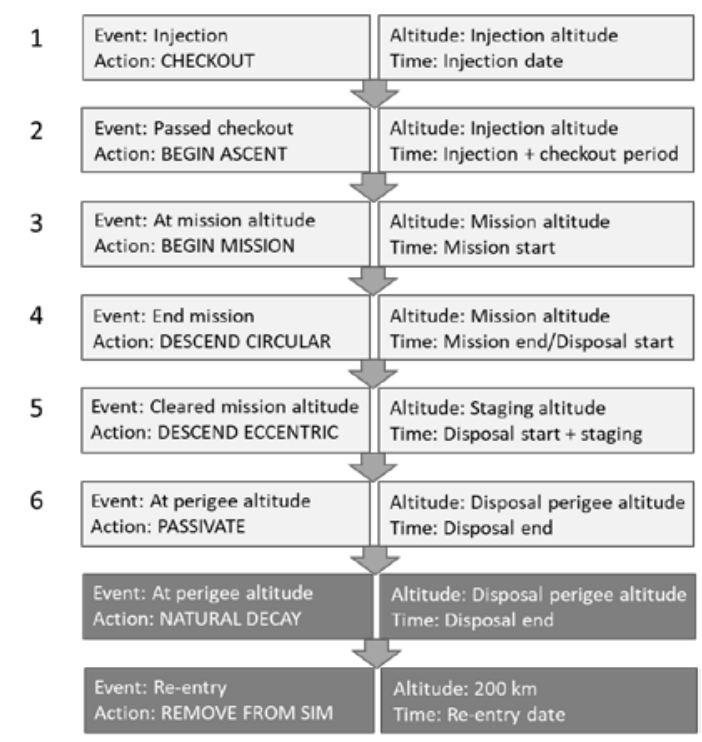

(a)

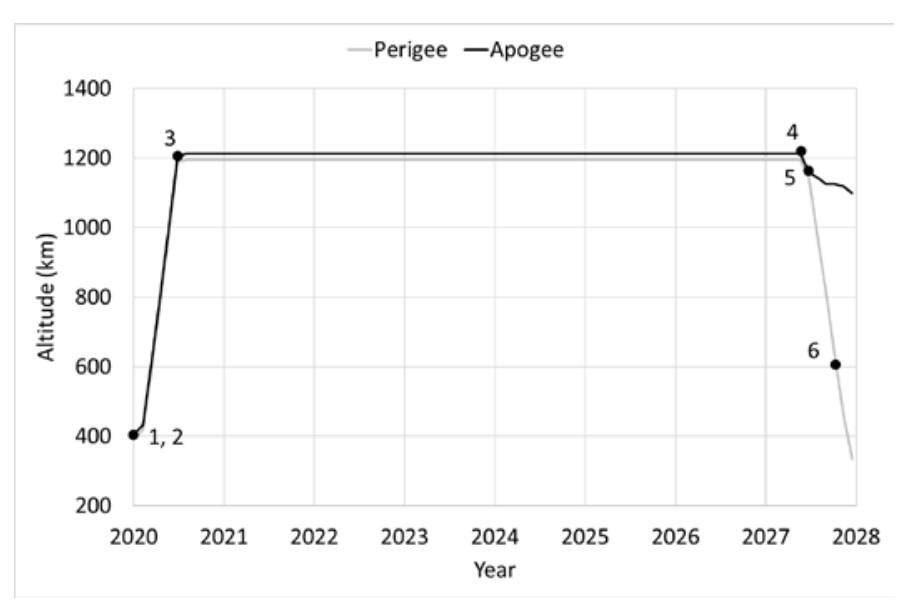

(b)

Fig. 1. Waypoints used to define the Concept of Operations for constellation spacecraft (a) and an example of their implementation on the evolution of the orbit of a spacecraft with a low-thrust propulsion system and a mission altitude of $1200 \mathrm{~km}$ (b). The bottom two entries in (a) represent phases in the lifetime where the spacecraft is not under active control. 
Tab. 1. Constellation parameters.

\begin{tabular}{lccccc}
\hline Component & $\begin{array}{c}\text { Number of } \\
\text { spacecraft }\end{array}$ & $\begin{array}{c}\text { Number of } \\
\text { orbital } \\
\text { planes }\end{array}$ & $\begin{array}{c}\text { Mission } \\
\text { altitude }(\mathrm{km})\end{array}$ & $\begin{array}{c}\text { Inclination } \\
\text { (deg.) }\end{array}$ & $\begin{array}{c}\text { Disposal } \\
\text { staging } \\
\text { altitude }(\mathrm{km})\end{array}$ \\
\hline VLEO A & 2400 & 40 & 345 & 42 & 300 \\
VLEO B & 2400 & 40 & 350 & 48 & 300 \\
VLEO C & 2400 & 40 & 355 & 52 & 300 \\
MLEO & 1800 & 30 & $1100 / 550$ & 54 & $1050 / 500$ \\
HLEO A & 480 & 8 & 1150 & 72 & 1050 \\
HLEO B & 480 & 8 & 1200 & 80 & 1050 \\
HLEO C & 480 & 8 & 1250 & 70 & 1050 \\
\hline
\end{tabular}

Here, 100 future projections from 1 February 2018 to 1 February 2218 were performed using DAMAGE, for each of the following scenarios:

1. With the MLEO component at $1100 \mathrm{~km}$ altitude;

2. With the MLEO component at $1100 \mathrm{~km}$ altitude and with orbital plane separation (OPS);

3. With the MLEO component at $1100 \mathrm{~km}$ and with satellite cross-sectional area at 4 square meters;

4. $\quad$ With the MLEO component at $550 \mathrm{~km}$ altitude;

5. With the MLEO component at $550 \mathrm{~km}$ altitude and 5\% post-mission disposal success rate for spacecraft in this component.

Although scenario 3 did not directly address the influence of orbit design, it was felt that understanding the implications of a reduction in the cross-sectional area of the constellation satellites in the MLEO component would provide a useful point of comparison for the other scenarios.

\section{RESULTS AND ANALYSIS}

With respect to the evolution of the number of trackable objects in LEO (Fig. 2) the DAMAGE results show that adding the large constellation always increased the orbital debris population by the end of the projection period, and always increased the population growth rate, regardless of the orbits used for the MLEO component. This is also in spite of robust mitigation measures related to post-mission disposal and the prevention of explosions. In other words, debris mitigation measures, including those based on orbit design, were unable to remove the long-term consequences of this constellation deployment within the simulation. In all other respects, the behavior of the population follows the three phases previously reported and associated with the constellation build-up, constellation disposal and growth due to fragmentations involving failed constellation spacecraft. By the year 2072, only constellation spacecraft that had failed to de-orbit from the HLEO components and the MLEO component (at 1100 $\mathrm{km}$ ) remained in the simulations. 


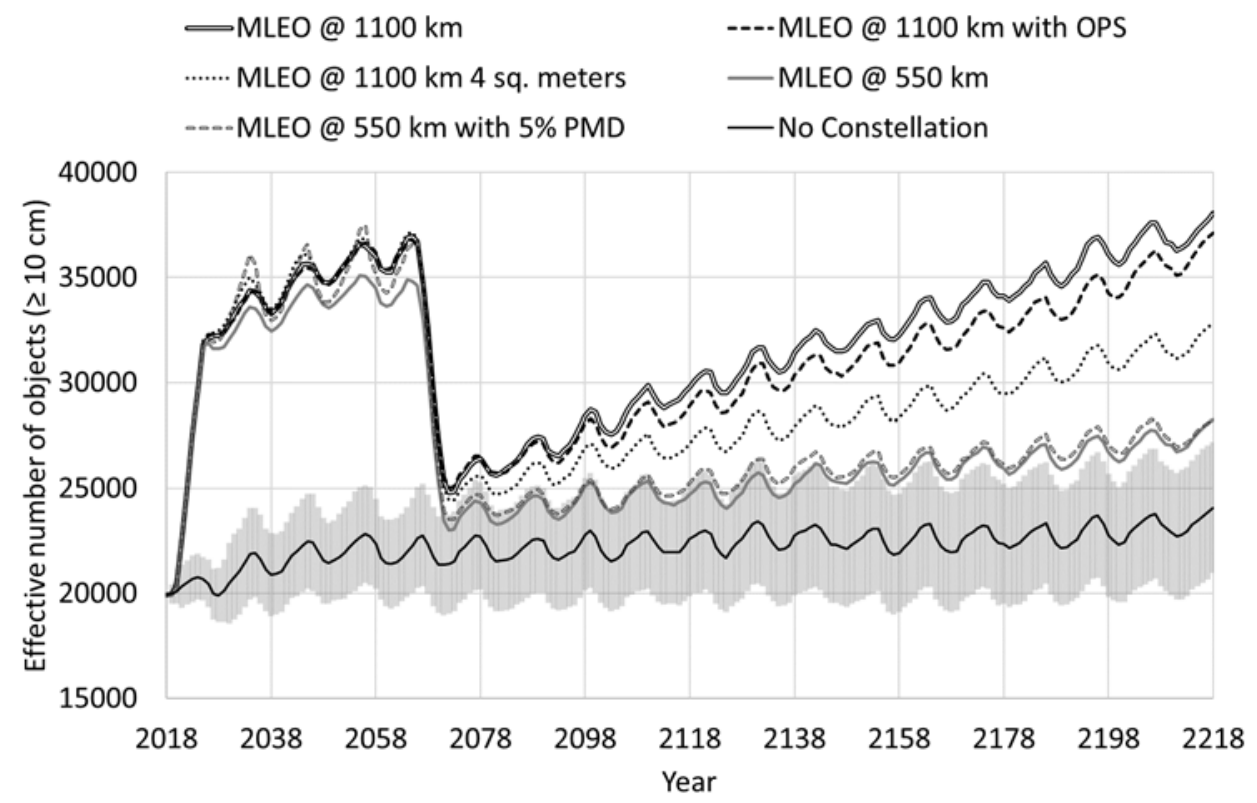

Fig. 2. Evolution of the $\geq 10 \mathrm{~cm}$ population in LEO for the study scenarios. The filled area represents the \pm 1 -sigma error for the no-constellation case.

With the MLEO component at $1100 \mathrm{~km}$ altitude, the average number of trackable objects was $58 \%$ higher than the no constellation case at the end of the projection period. Adding orbital plane separation to the MLEO component at $1100 \mathrm{~km}$ reduced the impact on the population by a relatively small amount (53\% higher than the no constellation case). Changing the design of the spacecraft in the MLEO component such that the cross-sectional area was onethird of the initial size resulted in a substantial benefit in terms of the final population size ( $36 \%$ higher than the no constellation case). However, changing the orbital altitude of the MLEO component from $1100 \mathrm{~km}$ to $550 \mathrm{~km}$ resulted in the greatest reduction in the population, to just $17 \%$ more trackable objects than the no constellation case.

The DAMAGE results also show that moving the MLEO component from $1100 \mathrm{~km}$ to $550 \mathrm{~km}$ altitude also appeared to add a high degree of resilience to spacecraft failures. In the case where only $5 \%$ of the constellation spacecraft in the MLEO component completed post-mission disposal maneuvers, the evolution of the LEO trackable population was essentially indistinguishable from the case where $95 \%$ of the spacecraft completed those maneuvers. Nevertheless, there was still an impact on the LEO environment in terms of the collision activity (Fig. 3 ). When the post-mission disposal success rate was very low, $14 \%$ more catastrophic collisions occurred on average than the equivalent case with high post-mission disposal success rate. However, moving the MLEO element from $1100 \mathrm{~km}$ to $550 \mathrm{~km}$ still had the greatest benefit of all the options considered, regardless of the post-mission disposal. In the best case (MLEO component at $550 \mathrm{~km}$ with 95\% post-mission disposal success rate), the total number of catastrophic collisions was $23 \%$ higher than the no constellation case by the end of the projection period, on average. The orbit design had the greatest impact at altitudes between $900 \mathrm{~km}$ and $1200 \mathrm{~km}$, where the constellation dominated the collision activity in all scenarios except when the MLEO component was at $550 \mathrm{~km}$ altitude (Fig. 4). Even so, some measures provided benefits when the MLEO component remained at $1100 \mathrm{~km}$ : decreasing the cross-sectional area of the MLEO spacecraft resulted in 58\% fewer catastrophic collisions at $1100 \mathrm{~km}$, whilst adding orbital plane separation resulted in $23 \%$ fewer catastrophic collisions at $1100 \mathrm{~km}$. 


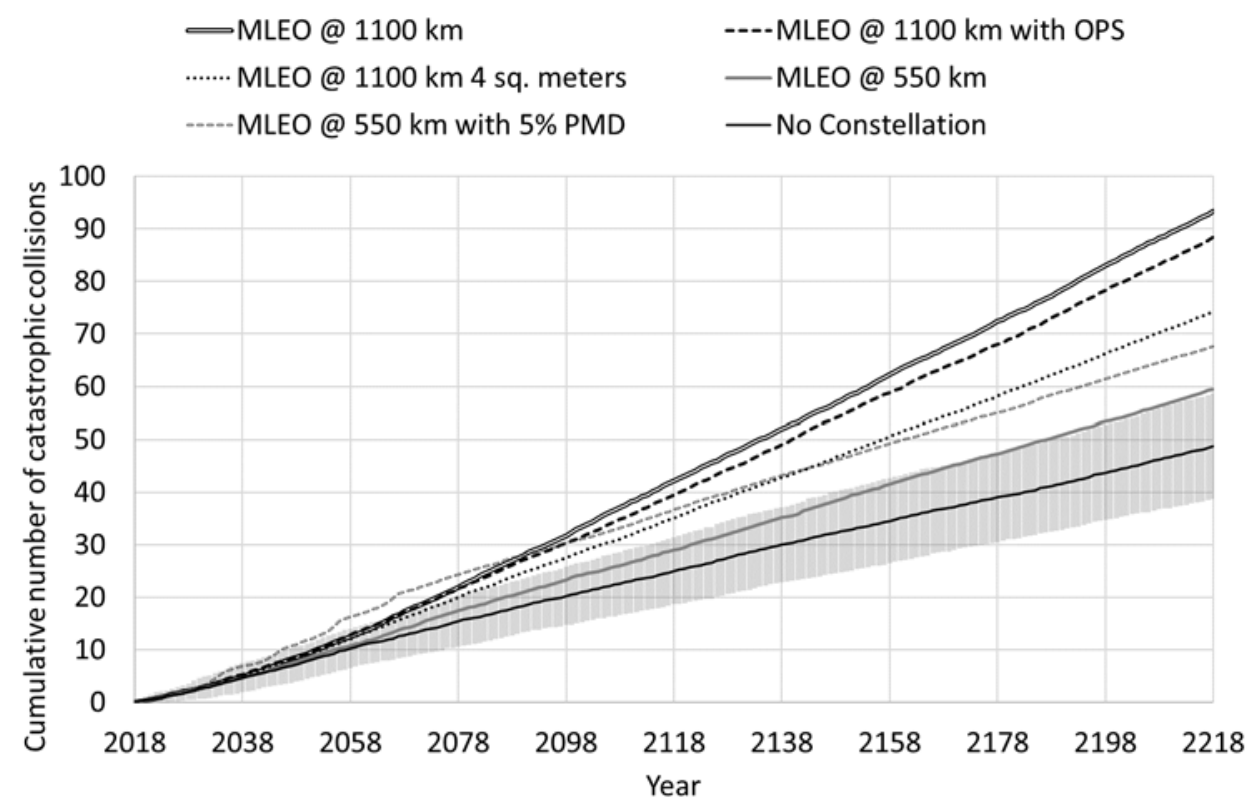

Fig. 3. Evolution of the cumulative number of catastrophic collisions for the study scenarios. The filled area represents the \pm 1 -sigma error for the no-constellation case.

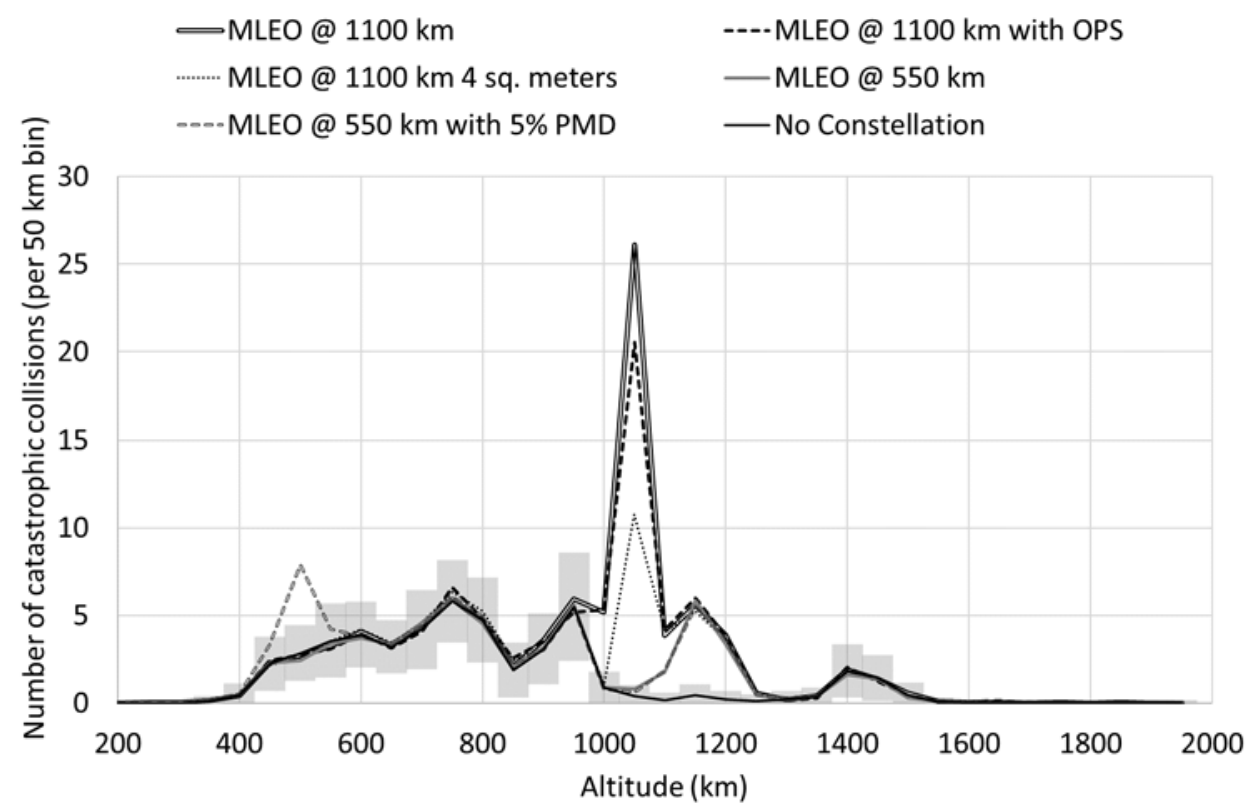

Fig. 4. Total number of catastrophic collisions with respect to altitude for the study scenarios. The filled area represents the \pm 1 -sigma error for the no-constellation case. 
When the MLEO component resided at $550 \mathrm{~km}$ altitude and the spacecraft performed fewer disposal maneuvers, there was an increase in the number of catastrophic collisions at $550 \mathrm{~km}$ and below, compared with the equivalent case for a high number of disposal maneuvers (Fig. 4). Intuitively, additional collisions would be expected to occur here, but in all other respects, the distribution of the catastrophic collisions is the same as for the case with the high maneuver rate. Taking the results from Fig. 2, Fig. 3 and Fig. 4 together, the conclusion is that a poor disposal maneuver rate will lead to a greater number of catastrophic collisions. However, if those occur at low altitude (e.g. $550 \mathrm{~km}$ ) then there will be little impact on the long-term evolution of the trackable population in LEO (Fig. 2) because the resulting fragments will decay rapidly once the constellation has ceased operations. Nonetheless, the additional collision fragments will lead to an elevated safety risk at or below $550 \mathrm{~km}$ while the constellation is operating. The elevated risk will translate directly into a disproportionately high number of collision avoidance maneuvers (CAMs) for all spacecraft residing in or crossing this region. Consequently, there is still an incentive for constellation operators to aim for a high post-mission disposal reliability even at relatively low altitudes, as this will reduce the CAM burden.

The spatial density distributions observed for each scenario at the end of the projection period are shown in Fig. 5. The results show that the constellation disrupted the spatial density at all altitudes in LEO above $700 \mathrm{~km}$, regardless of the scenario. The best outcome was achieved when the MLEO component was at $550 \mathrm{~km}$ altitude and similar outcomes were observed regardless of the post-mission disposal behavior. Nevertheless, separating the orbital planes or reducing the cross-sectional area of the spacecraft when the MLEO component was at $1100 \mathrm{~km}$ still gave some benefit.

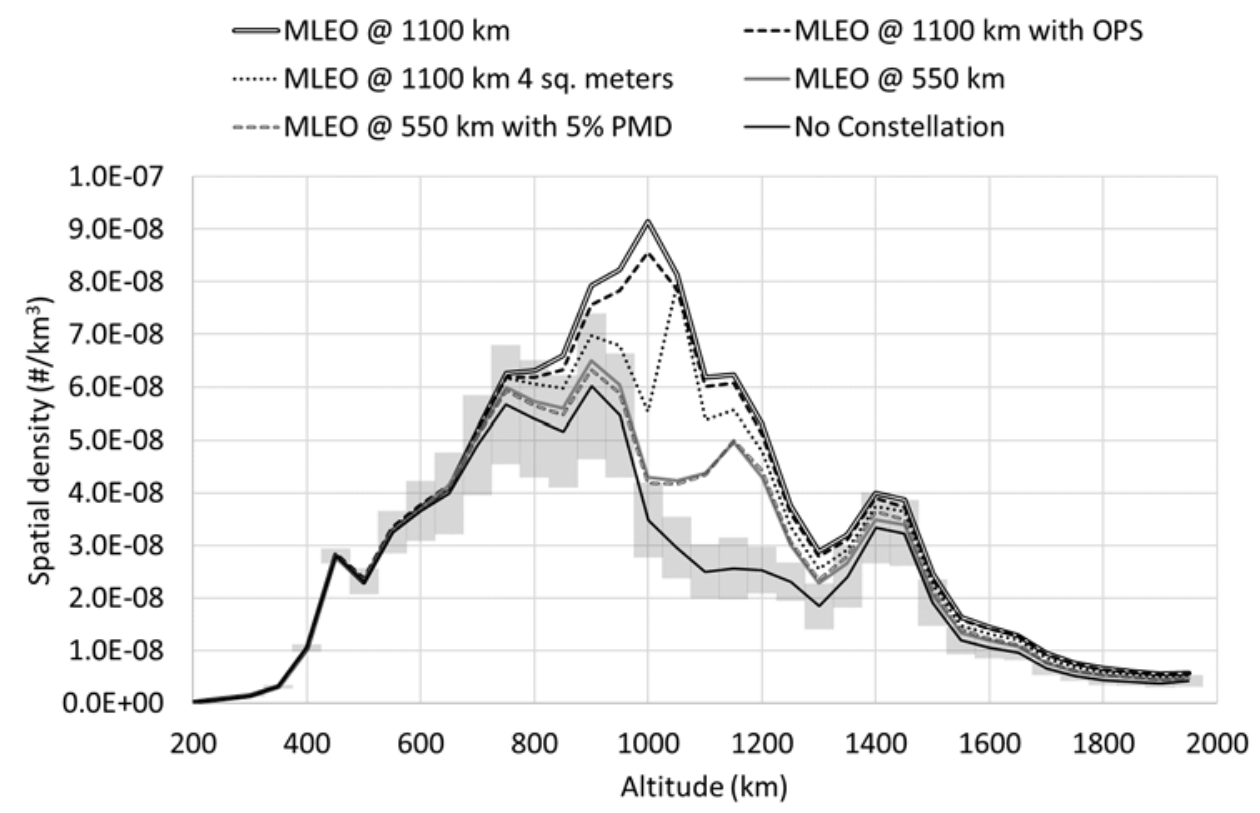

Fig. 5. Debris spatial density at the end the projection period for the study scenarios. The filled area represents the \pm 1 -sigma error for the no-constellation case. 


\section{CONCLUSIONS}

The study described in this paper aimed to understand the potential impacts on the LEO space environment of deploying a large constellation, comprising 10,440 spacecraft. Additionally, the study was designed to evaluate mitigation measures based on variations in the spacecraft orbits. A series of simulation studies, performed using the DAMAGE code, was used to address these objectives. The results show that for a constellation with this arrangement and number of spacecraft, there is a high likelihood of impacts on the debris population even if mitigation measures are adopted. However, the study results did demonstrate that moving a relatively small proportion (approximately 20\%) of the total constellation fleet from $1100 \mathrm{~km}$ to $550 \mathrm{~km}$ reduced the number of trackable objects in LEO by more than one-quarter, by the end of a 200-year projection period. At the same time, this orbit change also resulted in a substantial benefit with respect to the required post-mission disposal reliability. Even with a very low number of disposal maneuvers, there was little impact on the orbital debris population over the long-term. However, the poor post-mission disposal success rate did lead to enhanced collision activity near the 550 $\mathrm{km}$ altitude region for the life of the constellation. This collision activity would lead to a disproportionate increase in collision avoidance maneuvers for all spacecraft operating in or passing through this region.

The results also suggested that separating the orbital planes within the constellation could also provide some limited benefit with respect to the environment if spacecraft remained at a high altitude. In this case, further benefits could be achieved if the spacecraft design was such that the average cross-sectional area were reduced. No additional benefit resulted from this particular design change when the spacecraft were operated at a low altitude.

The study results confirm the benefits predicted by SpaceX, following the recent re-design of the Starlink constellation to accommodate the move of approximately 1600 spacecraft from $1100 \mathrm{~km}$ to $550 \mathrm{~km}$. In addition, the same benefits can be expected if the Amazon Kuiper constellation design remains unchanged and is deployed at 550 $\mathrm{km}$ altitude. However, the enhanced collision activity below $550 \mathrm{~km}$, arising from the inevitable failure of spacecraft from these constellations, is the trade-off that arises from improving the sustainability, and remains a concern. Consequently, additional work is required to better understand the collisional landscape that will be generated following the deployment of these large constellations and the burden it will place on all operators in terms of collision avoidance. Given the proximity of the International Space Station and the possibility of other activities associated with human spaceflight, it is imperative that this understanding is gained so that appropriate countermeasures can be implemented.

\section{ACKNOWLEDGEMENTS}

The author would like to thank colleagues from the Space Debris Office of the European Space Agency for the provision of and permission to use the MASTER population and launch traffic for this work.

\section{REFERENCES}

1. B. Bastida Virgili et al., Risk to space sustainability from large constellations of satellites, Acta Astronautica Vol. 126, pp. 154-162, 2016.

2. H.G. Lewis et al., Sensitivity of the space debris environment to large constellations and small satellites, J. British Interplanetary Society, Vol. 70 (2-4), pp. 105-117, 2017.

3. J. Radtke et al., Interactions of the space debris environment with mega constellations - using the example of the OneWeb constellation, Acta Astronautica, Vol. 131, pp. 55-68, 2017.

4. G.E. Peterson et al., Implications of proposed small satellite constellations on space traffic management and long-term growth in near-earth environment, 67th International Astronautical Congress, Guadalajara, Mexico, 2016.

5. B. Revelin and J.C. Dolado-Perez, Risk induced by the uncatalogued space debris population in the presence of large constellations, J. British Interplanetary Society, Vol. 70 (2-4), pp. 98-104, 2017.

6. J.-C. Liou, J. et al. “NASA ODPO’s Large Constellation Study”, Orbital Debris Quarterly News, Vol. 22 (3), pp. 4-7, 2018. 
7. Lewis, H.G., et al. "Long-Term Environmental Effects of Deploying the OneWeb Satellite Constellation”, $70^{\text {th }}$ International Astronautical Congress, Washington DC, USA, 2019.

8. Lewis, H.G. et al. "Mitigation Measures for Large Constellations," $68^{\text {th }}$ International Astronautical Congress, Adelaide, Australia, 2017. 